\title{
pH-induced Softening of Polyelectrolyte Microcapsules without
} Apparent Swelling

Miju Kim, ${ }^{\dagger}+$ Junsang Doh, **, $\S$ and Daeyeon Lee *广

$\dagger$ Department of Chemical and Biomolecular Engineering, University of Pennsylvania, Philadelphia, PA 19104, USA

\$ Department of Mechanical Engineering, Pohang University of Science and Technology (POSTECH), Pohang, Gyeongbuk 790-784, Republic of Korea

$\S$ School of Interdisciplinary Bioscience and Bioengineering (I-Bio), Pohang University of Science and Technology (POSTECH), Pohang, Gyeongbuk 790-784, Republic of Korea

*Address correspondence to daeyeon@seas.upenn.edu (Prof. Daeyeon Lee) and jsdoh@postech.ac.kr (Prof. Junsang Doh) 


\section{Experimental Section}

\section{Fabrication of (PAA/bPEI) NICE microcapsules}

3 Detailed methods regarding the fabrication of NICE microcapsules has been introduced in a

4 previous report. ${ }^{1}$ Briefly, to generate water-in-oil-in-water $(\mathrm{W} / \mathrm{O} / \mathrm{W})$ double emulsions that are

5 used as templated for NICE microcapsules, we use a glass-capillary microfluidic device that

6 combines co-flow and flow-focusing geometries. $0.1 \mathrm{wt} \%$ PAA dissolved in deionized water

$7 \quad(\mathrm{pH} 3.7)$ is used as the inner phase, and $0.1 \mathrm{wt} \% \mathrm{bPEI}$ dissolved in a mixture of chloroform

8 and hexane (Fisher Scientific) in a volume ratio of $1: 1$ containing $1 \mathrm{wt} \%$ sorbitan trioleate

9 (Sigma) is used as the middle phase. A $2 \mathrm{wt} \%$ poly (vinyl alcohol) (PVA, 87-90\% hydrolyzed, 10 average molecular weight: 30,000-70,000 g/mol, Sigma-Aldrich) solution is used as the outer 11 phase. To maintain the stability of NICE double emulsions and reliably induce the collection solution. After collection, we discard the supernatant containing separated oil droplets and exchange the outer solution with fresh $\mathrm{pH}$ 2-water multiple times.

Osmotic pressure-induced buckling method

The elasticity of the NICE microcapsule shell is determined using osmotic pressure-induced buckling. Before starting this experiment, (PAA/bPEI) NICE microcapsules are stored in $\mathrm{pH}$ 2-water $(10 \mathrm{mM} \mathrm{HCl})$ and washed with fresh $\mathrm{pH}$ 2-water several times. To observe microcapsules on a clear and flat surface using microscope, we use 24-well polystyrene plates having flat bottom (Corning). Each well is filled with $500 \mu \mathrm{L}$ of pure NICE microcapsule solution, and then sequentially filled with $500 \mu \mathrm{L}$ PEG solution to yield $1 \mathrm{~mL}$ solution with desired PEG concentration. After gentle mixing, the solutions containing NICE microcapsules are stored at room temperature for 2-4 hours. We visually inspect the optical microscopy images of microcapsules to detect the deformation of microcapsules under osmotic stresses. Because NICE microcapsules are relatively large $(\sim 100 \mu \mathrm{m})$, we can readily distinguish 
1 intact spherical microcapsules from those that have undergone buckling. At least 200

2 microcapsules are counted for each PEG concentration. To obtain the relationship between the

3 fraction of deformed capsules and PEG concentration, we test at least 10 different

4 concentration values. Analyses of graphs are performed using GraphPad software (GraphPad

5 Prism 5).The solution $\mathrm{pH}$ is adjusted by using $1 \mathrm{M} \mathrm{HCl}$ or $\mathrm{NaOH}$ solutions. NICE

6 microcapsules are stored in each $\mathrm{pH}$-adjusted solution for at least 2 days before being

7 subjected to osmotic shock. All experiments are performed at room temperature. 

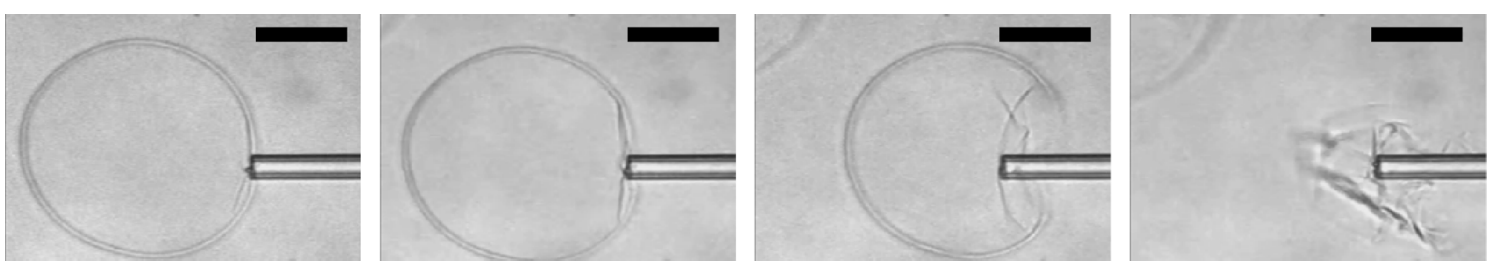

Figure S1. Optical microscopy images showing buckling-induced collapse of a NICE microcapsule under negative pressure applied by micropippette. All scale bars are $50 \mu \mathrm{m}$. 


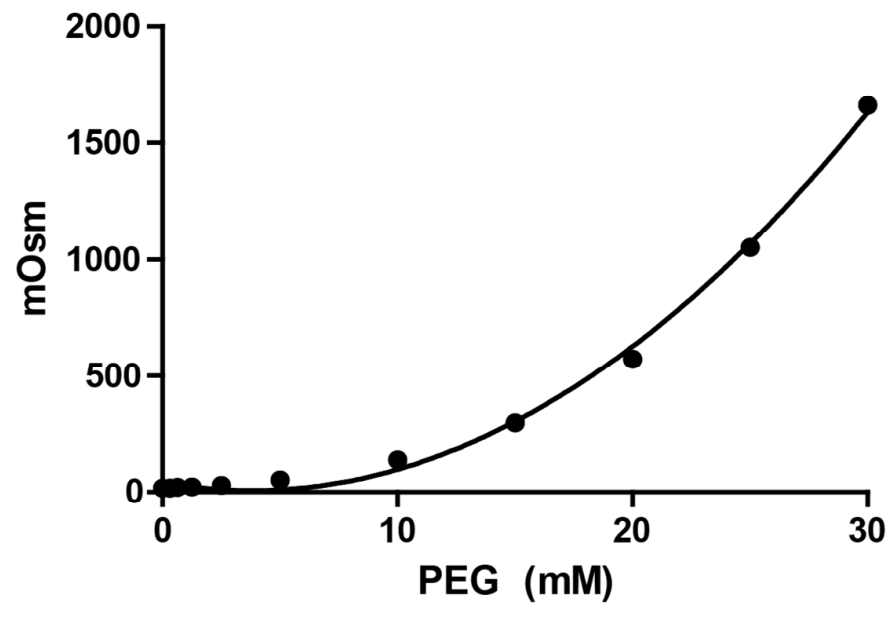

Figure S2. Osmolarity calibration curve for PEG (4000 $\mathrm{g} / \mathrm{mol})$ solutions of different concentrations. 


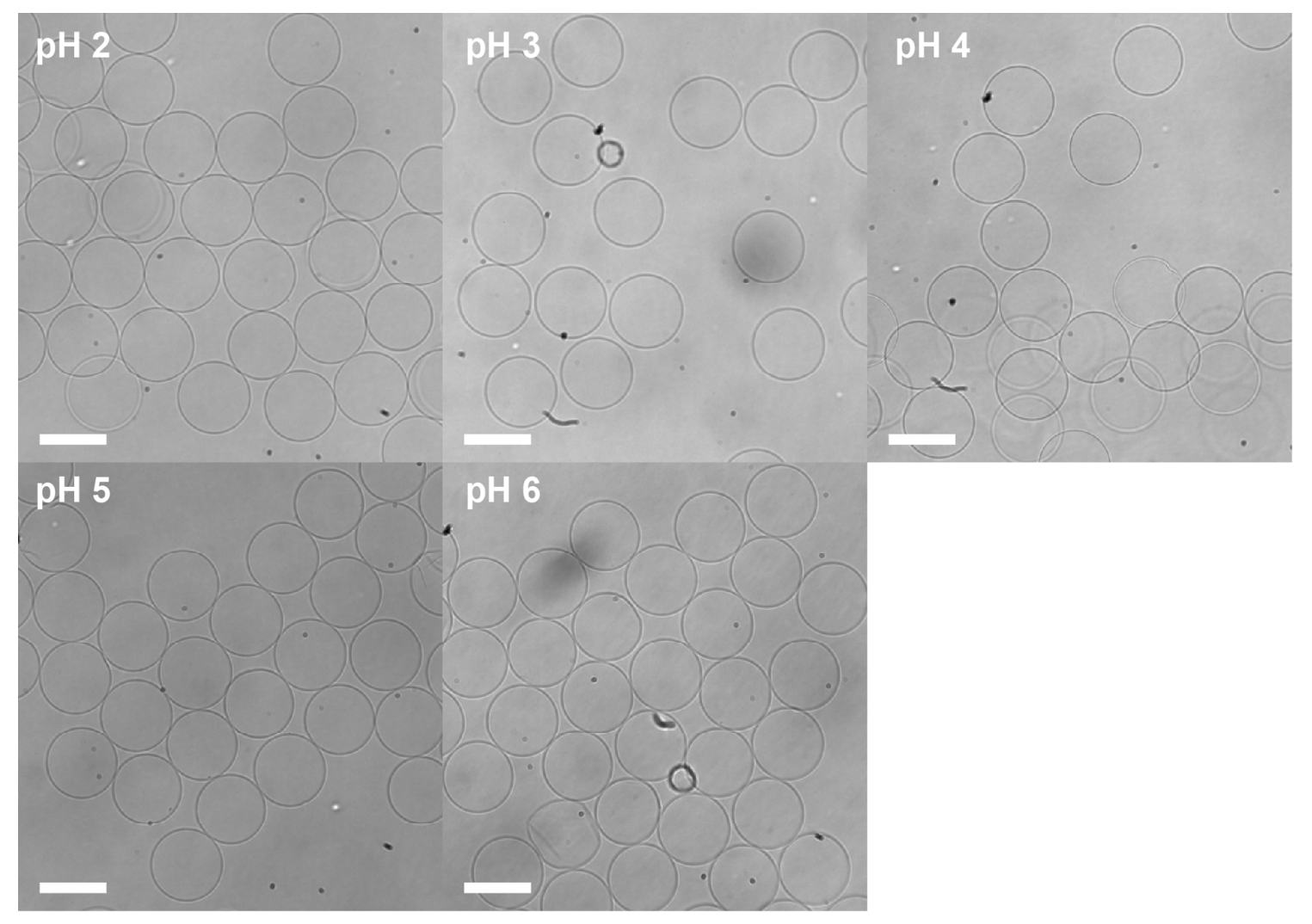

Figure S3. Optical microscopy images showing no detectable changes in the size of NICE microcapsules when the solution $\mathrm{pH}$ is increased from $\mathrm{pH} 2$ to 6 . All scale bars are $100 \mu \mathrm{m}$.

Rationalizing the use of dry shell thickness obtained at pH 2 to estimate the shell stiffness of NICE microcapsules at other pH conditions. If we assume that the shell thickness doubles with no apparent changes in the capsule radius as the solution $\mathrm{pH}$ if raised from 2.0 to 4.0, this would mean that the actual modulus would be $25 \%$ of our estimated value based on Equation 1. Thus our conclusion that the shell undergoes softening as the $\mathrm{pH}$ is raised from 2 to 6 is not affected. Since we assume that the shell thickness does not change, our estimation of shell stiffness obtained between $\mathrm{pH} 3$ and 6 represent the upper bound values. 


\section{Reference}

(1) Kim, M.; Yeo, S. J.; Highley, C. B.; Burdick, J. A.; Yoo, P. J.; Doh, J.; Lee, D., ACS nano 2015, 9 (8), 8269-8278. 

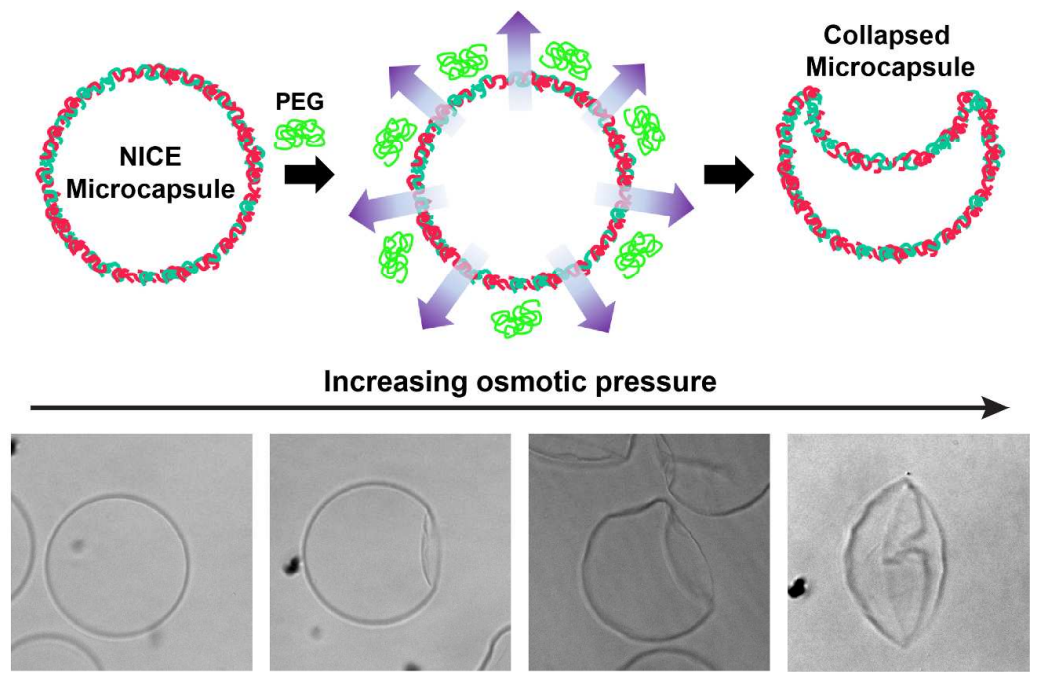

Table of contents 